\title{
Pop! Goes the weasel
}

\author{
Castigliano M. Bhamidipati, DO, PhD, MSc, and Nahush A. Mokadam, MD
}

\author{
From the Division of Cardiothoracic Surgery, University of Washington Medical Center, Seattle, Wash. \\ Disclosures: N.A.M. is an investigator for HeartWare (Medtronic), St Jude (Abbott), and SynCardia, and is a \\ consultant for HeartWare (Medtronic) and St Jude (Abbott). The other author has nothing to disclose with re- \\ gard to commercial support. \\ Received for publication Feb 8, 2017; accepted for publication Feb 15, 2017; available ahead of print March 23, \\ 2017. \\ Address for reprints: Nahush A. Mokadam, MD, University of Washington Medical Center, Division of Cardio- \\ thoracic Surgery, Department of Surgery, 1959 NE Pacific St, Box 356310, Seattle, WA 98195-6310 (E-mail: \\ mokadamn@uw.edu). \\ J Thorac Cardiovasc Surg 2017;153:1411-2 \\ $0022-5223 / \$ 36.00$ \\ Copyright (C) 2017 by The American Association for Thoracic Surgery \\ http://dx.doi.org/10.1016/j.jtcvs.2017.02.041
}

The pathobiology, management, and surveillance schema of saccular aneurysms in the thoracic aorta remain the subject of debate. We have long postulated that fusiform elongation and dilation of the aorta create an aneurysm in which laminar flow leads to forces that result in more uniform wall tension, whereas saccular aneurysms may have forces that give rise to disparate areas of wall tension. In the abdominal aorta, the association between morphology and rupture comes from historical observations of 2-dimensional angiograms. ${ }^{1}$ This was partially supported by a tortuosity index derived using 3dimensional computed tomography (CT) as a surrogate for increased turbulence and regional wall stress. ${ }^{2}$ Therefore, it is generally regarded that thoracic saccular aneurysms are more malignant. Unlike the abdominal aorta where saccular aneurysms are rarely encountered, in the thoracic aorta, especially in the region of the distal arch, ductus aneurysms are not infrequently diagnosed. ${ }^{3,4}$ Despite our intuition, the lack of good data makes the management and relative risk of rupture of saccular aneurysms in the thoracic aorta complex and poorly understood. ${ }^{5,6}$

Finite element analysis of saccular aneurysms has taught us that (1) a separation vortex occurs in the whole circumferential region of the aneurysm, (2) the separation vortex is distorted by the geometric shape of the aneurysm, and (3) 2 other vortexes induced by the separation vortex exist at the proximal and distal wall of aneurysms. ${ }^{7}$ How saccular aneurysms interplay with native collagen versus elastin concentrations of the aorta is unknown. Collectively, this has led to aggressive open, endovascular, and hybrid management of saccular aneurysms to ensure aneurysmal exclusion. ${ }^{6}$ As technology improves and our ability to manipulate raw imaging data evolves, more information will be learned. Fourdimensional (4D)-flow magnetic resonance imaging (MRI) and computational fluid dynamic simulations evaluating aortic flow dynamics are already important to cerebral aneurysms and aortic valve configurations. Separately, we also know that wall shear stress (WSS) and oscillatory shear index are important to cerebral aneurysms and abdominal aortic aneurysms. Positron emission tomography scans and

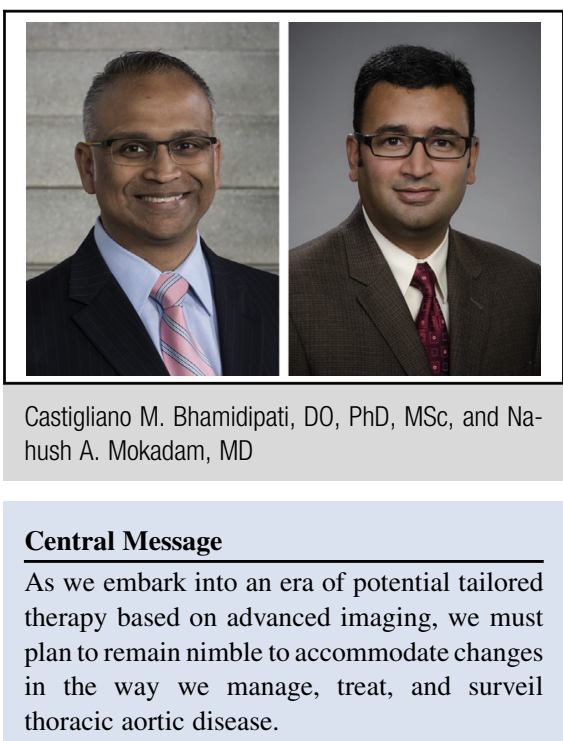

See Article page 1413 .

18-fluorodeoxyglucose uptake have been shown to be a significant imaging biomarker in abdominal aortic aneurysms. Collectively, these types of data are sparse for the thoracic aorta, much less so for discriminating rupture risk between fusiform and saccular aneurysms.

In this issue of the Journal, Natsume and colleagues ${ }^{4}$ provide what could become seminal work in the study of saccular aneurysms of the proximal aorta. They analyzed the geometry of aneurysms using multiplanar reconstruction of CT, and they evaluated WSS and vortex flow using 4D-flow MRI. Their results suggest that flow dynamics of saccular and fusiform aneurysms differ and that saccular aneurysms of the outer curvature behave more fusiform than of the inner curvature. Although we may not have direct observational studies from large groups of patients with saccular aneurysms, we may finally have evidence supporting our clinical suspicions!

For the first time, the management of saccular thoracic aneurysms can be driven by objective metrics. Our decision making when contemplating management can be more informed and measured. Although 4D-flow MRI is costly and time-consuming (which makes this application currently impractical), sac depth/neck width ratio can readily be calculated from any contrast-enhanced CT. For patients with saccular aortic aneurysms, this ratio can be used to estimate their risk of rupture. As concluded in the article, when this ratio is less than 0.8 , using diameter measurements alone may be appropriate for observation; 
however, when this ratio is greater than 0.8 , a more aggressive operative approach may be warranted regardless of size. This provides additional fidelity to the Joint Council of the Society for Vascular Surgery and the International Society for Cardiovascular Surgery on their recommendation of saccular aneurysms being a surgical indication independent of size.

As we strive to address these aneurysms before the development of complications or rupture, we still have questions moving forward: What is the real natural history of saccular aneurysms? Should everyone receive a 4D-flow MRI? Should we measure WSS and oscillatory shear index on all patients with an aneurysm? Perhaps most pressingly, how should we currently counsel patients when we measure sac depth/neck width ratio greater than 0.8 from their contrast-enhanced CT?

As we embark into an era of potential tailored therapy, we must plan to remain nimble to accommodate changes in the way we manage, treat, and surveil thoracic aortic disease.

\section{References}

1. Szilagyi DE, Smith RF, DeRusso FJ, Elliott JP, Sherrin FW. Contribution of abdominal aortic aneurysmectomy to prolongation of life. Ann Surg. 1966;164: 678-99.

2. Pappu S, Dardik A, Tagare H, Gusberg RJ. Beyond fusiform and saccular: a novel quantitative tortuosity index may help classify aneurysm shape and predict aneurysm rupture potential. Ann Vasc Surg. 2008;22:88-97.

3. Taylor BV, Kalman PG. Saccular aortic aneurysms. Ann Vasc Surg. 1999;13: 555-9.

4. Natsume K, Shiiya N, Takehara Y, Sugiyama M, Satoh H, Yamashita K, et al. Characterizing saccular aortic arch aneurysms from the geometryflow dynamics relationship. $J$ Thorac Cardiovasc Surg. 2017;153: 1413-20.

5. Criado FJ. Aneurysm morphology matters: fusiform vs. saccular. J Endovasc Ther. 2013;20:207-9.

6. Preventza O, Coselli JS. Saccular aneurysms of the transverse aortic arch: treatment options available in the endovascular erabased on a presentation at the 2013 VEITH Symposium, November 19-23, 2013 (New York, NY, USA). Aorta (Stamford). 2015;3:61-6.

7. Matsuzawa T. Finite element analysis in three-dimensional flow through a lateral saccular aneurysm. Front Med Biol Eng. 1993;5:89-94.

8. Kotze CW, Menezes LJ, Endozo R, Groves AM, Ell PJ, Yusuf SW. Increased metabolic activity in abdominal aortic aneurysm detected by $18 \mathrm{~F}$-fluorodeoxyglucose (18F-FDG) positron emission tomography/computed tomography (PET/CT). Eur J Vasc Endovasc Surg. 2009;38:93-9. 